\title{
Pragmatic aspects of uncertainty propagation: A conceptual review
}

\author{
W. Carlisle Thacker*, Mohamad Iskandarani ${ }^{\mathrm{a}}$, Rafael C. Gonçalves ${ }^{\mathrm{a}}$, \\ Ashwanth Srinivasan ${ }^{\mathrm{b}}$, Omar M. Knio ${ }^{\mathrm{c}, \mathrm{d}}$ \\ ${ }^{a}$ University of Miami, Miami, FL \\ ${ }^{b}$ Tendral Corporation, Miami, FL \\ ${ }^{c}$ Duke University, Durham, $N C$ \\ ${ }^{d}$ King Abdullah University of Science and Technology, Saudi Arabia
}

\begin{abstract}
When quantifying the uncertainty of the response of a computationally costly oceanographic or meteorological model stemming from the uncertainty of its inputs, practicality demands getting the most information using the fewest simulations. It is widely recognized that, by interpolating the results of a small number of simulations, results of additional simulations can be inexpensively approximated to provide a useful estimate of the variability of the response. Even so, as computing the simulations to be interpolated remains the biggest expense, the choice of these simulations deserves attention. When making this choice, two requirement should be considered: (i) the nature of the interpolation and ii) the available information about input uncertainty. Examples comparing polynomial interpolation and Gaussian process interpolation are presented for three different views of input uncertainty.
\end{abstract}

\footnotetext{
${ }^{*}$ Corresponding author

Email addresses: carlisle.thacker@gmail.com (W. Carlisle Thacker), miskandarani@rsmas.miami .edu (Mohamad Iskandarani), rgoncalves@rsmas.miami.edu (Rafael C. Gonçalves), a.srinivasan@tendral .com (Ashwanth Srinivasan), omar.knio@duke.edu (Omar M. Knio) 
Keywords: polynomial chaos, Gaussian process, optimal interpolation, ocean modeling, error propagation, uncertainty quantification

\section{Introduction}

tative predictions of future conditions. For the value of such predictions to be assessed, they should be accompanied by quantitative information about their reliability.

While the model's formulation - the physical processes and how they are treated - has a major impact on its prediction, the impact of alternative formulations is not discussed here ${ }^{1}$. Instead, the model is regarded as a reliable black box and the focus is on assessing the uncertainties of the numbers it produces (its outputs or responses), which result from the uncertainties of the numbers it is given (its inputs). When making planning decisions, only a few of a model's many outputs are generally of interest, so estimates of accuracy can be restricted to those few outputs. Computational resources limit the number of uncertain inputs that can be treated simultaneously to a manageable few, so most of the inputs must be treated as known even though their values are by no means certain. What we discuss here should be regarded as conditional uncertainties - uncertainties conditioned on the values assumed to be known and on the model's formulation. We are interested in how the model transforms information about the uncertainty of selected inputs into

\footnotetext{
${ }^{1}$ Webster and Sokolov (2000) discuss the role of model formulation when quantifying uncertainty of climate projections.
} 
information about key outputs.

Suppose uncertainties of the inputs are quantified as a probability density centered on their most likely values, and suppose that a large ensemble of possible inputs are sampled according to this density. Then, assuming sufficient computational resources are available, for each set of inputs from this ensemble the model can be run to provide corresponding responses, and a histogram of these responses can approximate the probability density quantifying the uncertainty of the model's response stemming from the uncertainty of its inputs. In order to carry out this Monte Carlo agenda, two practical issues must be addressed: (1) how to proceed when the model's computational requirements limit the number of runs that can be made and (2) how to proceed when there is limited information about the uncertainties of the models inputs ${ }^{2}$.

Before computers were available propagating uncertainty had already been recognized as being important. Wiener (1938) addressed the problem within the context of a single uncertain input and a single response. Rather than addressing issue (2), he assumed the input density to be a known Gaussian. His approach was to approximate the response as a polynomial function of the input expressed as a truncated expansion in Hermite polynomials ${ }^{3}$, and solving for the expansion coefficients became the major computational

\footnotetext{
${ }^{2}$ Webster and Sokolov (2000) discuss the issue of uncertainty about input uncertainty.

${ }^{3}$ His use of Hermite polynomials was tied to his use of a Gaussian probability density, since Hermite polynomials are orthogonal when weighted by a Gaussian density. If he had expanded in Legendre polynomials, the method requires a uniform input density, and Laguerre polynomials require an exponential density. See, for example, Eldred et al. (2008) or Xiu (2009).
} 
task. ${ }^{4}$. Because of the orthogonality of Hermite polynomials when weighted by the Gaussian density ${ }^{5}$, statistics such as the mean and variance of the response could be expressed as simple functions of the expansion coefficients, so there was no need to sample from the input density. Wiener's approach has spawned a great deal of recent activity and is referred to as polynomial chaos in the engineering literature ${ }^{6}$.

Although Wiener (1938) did not appear to recognize it, the most important aspect of his approach was approximating a nonlinear response using an inexpensively evaluated polynomial function, which could serve as an emulator or surrogate for the original model. Because running the emulator is much less expensive than running the original model, Monte Carlo sampling becomes affordable. This reduces issue (1) to the more manageable concern of how using emulated responses impacts our view of response uncertainty. Moreover, it also provides an answer to issue (2) - how to deal with uncertainty about input uncertainty: simply consider several alternative descriptions of the input uncertainty and construct histograms of the response for each. Comparing the histograms to assess the impacts of the the alternative views of input uncertainty clearly must reflect how they alter practical decisions.

Running the emulator is simple. The bulk of the computation effort is in building it, as the model must be run repeatedly to generate enough responses to interpolate. A major point of this paper is that it is best to organize

\footnotetext{
${ }^{4}$ See Appendix A for a brief discussion of how the coefficients might be computed. ${ }^{5}$ Recall its use in the context of the meridional structure of equatorial Rossby waves.

${ }^{6}$ The reviews of Najm (2009) and Xiu (2009) provide brief introductions to polynomial chaos and references to much of its literature.
} 
computational effort in such a way that allows alternative input densities to be explored in a flexible and cost-effective manner. For each input density, sampling should reflect its more likely values, so that the response is most accurate where it is most important. When using quadrature to compute the expansion coefficients, the polynomial chaos formulation that links the input density to the orthogonality of the polynomials guarantees this sort of sampling as long as the input density is correct, but changing the input density requires redoing the sampling ${ }^{7}$. Our point is that it is important to be able to interpolate more flexibly so that sampling and interpolating can be incremental, so that as the view of input uncertainty changes existing samples need only be supplemented by a few more to improve accuracy for input values previously considered unlikely. Solving an algebraic system for the expansion coefficients, rather than using quadrature, provides this needed flexibility ${ }^{8}$.

An emulator need not be restricted to polynomial interpolation. Gaussian process interpolation has also been used for this purpose ${ }^{9}$. Rather than treating the response as a sum of specified functions of the random inputs, the response function itself is regarded as a random function. More specifically, for each input, the output has a specified mean and variance, and for each pair of inputs there is a specified covariance. It is easy to recognize

\footnotetext{
${ }^{7}$ See Appendix B.

${ }^{8}$ See Appendix C.

${ }^{9}$ Rougier et al. (2009) have used Gaussian processes for characterizing responses of climate models. See also the articles by Sacks et al. (1989) and by Kennedy and O'Hagan (2000) and the excellent book by Rasmussen and Williams (2006). Appendix D provides a brief discussion of Gaussian process interpolation. A reviewer recommended the folowing articles as potentially inspiring: French (2003); Ratto et al. (2009); Yang (2011); Borgonovo et al. (2012); Castaings et al. (2012).
} 
this as a novel application of optimal interpolation, as it corrects a prior description of the response surface using an assumed covariance function to interpolate the information provided by model simulations. Using Gaussian processes shifts the focus from determining coefficients of a polynomial approximation to choosing appropriate mean and covariance functions. Once these choices have been made and the responses of the exploratory ensemble have been assimilated, the updated mean function serves as the approximate response function and the updated variance function provides a measure of its accuracy.

We use a single input and a single output for illustrating these ideas, as that allows the underlying issues to be discussed more clearly. To illustrate our points, we use a model that simulates the fate of oil droplets emerging from a deep underwater source and rising due to their buoyancy as they are advected by a prescribed velocity field. This model was chosen because a database of its simulations was available and new simulations could be avoided. Uncertainty in droplet size is the single uncertain input of interest and the single response is the surface concentration of oil in a region some distance from the source. The details of this model are unimportant here. What matters is that the response is a highly nonlinear function of the input. In the examples discussed below, polynomials and Gaussian processes provide alternative interpolations for the same sets of simulated responses and thus a framework in which the separate roles of the choice of input density and the choice of the interpolation method can be addressed.

These simulations had previously been used at an early stage of model development to check how the uncertainty of surface oil concentration within 
a restricted region depended on the uncertainty of droplet size at the spill site. These simulations are from two different quadrature ensembles, one providing a 6th-degree polynomial approximation to the response function and the other providing a 20th-degree polynomial approximation, which might be regarded as being exact over the range of inputs considered. When combined and then sub-sampled they provide the possibility of exploring different approaches to interpolation and different views of input uncertainty.

This paper takes a step-by-step approach to illustrate the above ideas incrementally. Section 2 describes the data from the database of quadrature simulations and discusses the differences in the resulting 6th- and 20th-degree polynomial approximations to the response curve, both of which indicate that the response is a highly nonlinear function of droplet size. Section 3 discusses the histograms characterizing the uncertainty in surface-concentration response, which reflect the differences between these two views of the response curve, under the assumption that the uniform probability density for droplet size on which the quadrature ensembles were based is the correct density. Section 4 illustrates how the existing 20th-degree polynomial approximation to the response can be used to assess the uncertainty of the surface oil concentration when the initial assumption of uniform probability of droplet size is replaced with alternate assumptions - without the need of any new simulations. Section 5 illustrates how decoupling the polynomial approximation from the input probability density can provide a more flexible choice of simulations. And section 6 explores Gaussian process interpolation as an alternative to polynomial interpolation and illustrates its impact on the propagation of uncertainty from droplet size to surface concentration. As the focus is on 
practicality, methodological details are confined to appendices. The paper ends with a few concluding remarks.

\section{Simulated data}

The context of this study is provided by a model being constructed for simulating the effects of the Deepwater Horizon oil spill in the Gulf of Mexico. While that model has many uncertain inputs and many responses worthy of study, here we focus on one particular uncertain input and one particular response, as they had been examined in the early stages of model construction and those quadrature simulations offered a convenient context for this study. The uncertain input is the size of the oil droplets at the trap height where the droplets accumulate at the site of the spill ${ }^{10}$, and the uncertain output is the concentration of oil at the sea surface in a region some distance from the spill site.

For illustrating the separate roles of the approximate response function and the assumed nature of the input's uncertainty, a detailed description of the model that transforms droplet size to surface oil concentration is unnecessary. It is sufficient to know that the droplets are injected at depth and are carried by spatially and temporally varying eddies and currents driven by meteorological forcing as they rise to the surface due to their size-dependent buoyancy. Thus, the response function for the region reflects the itinerary taken by each droplet on its way to the surface of the study region at a

\footnotetext{
${ }^{10}$ In actuality, there is a distribution of droplet sizes and the parameters characterizing the shape of this distribution might be taken as uncertain inputs, but for simplicity all droplets were taken to have the same size and the uncertainty of the size provided a proxy for a size distribution.
} 


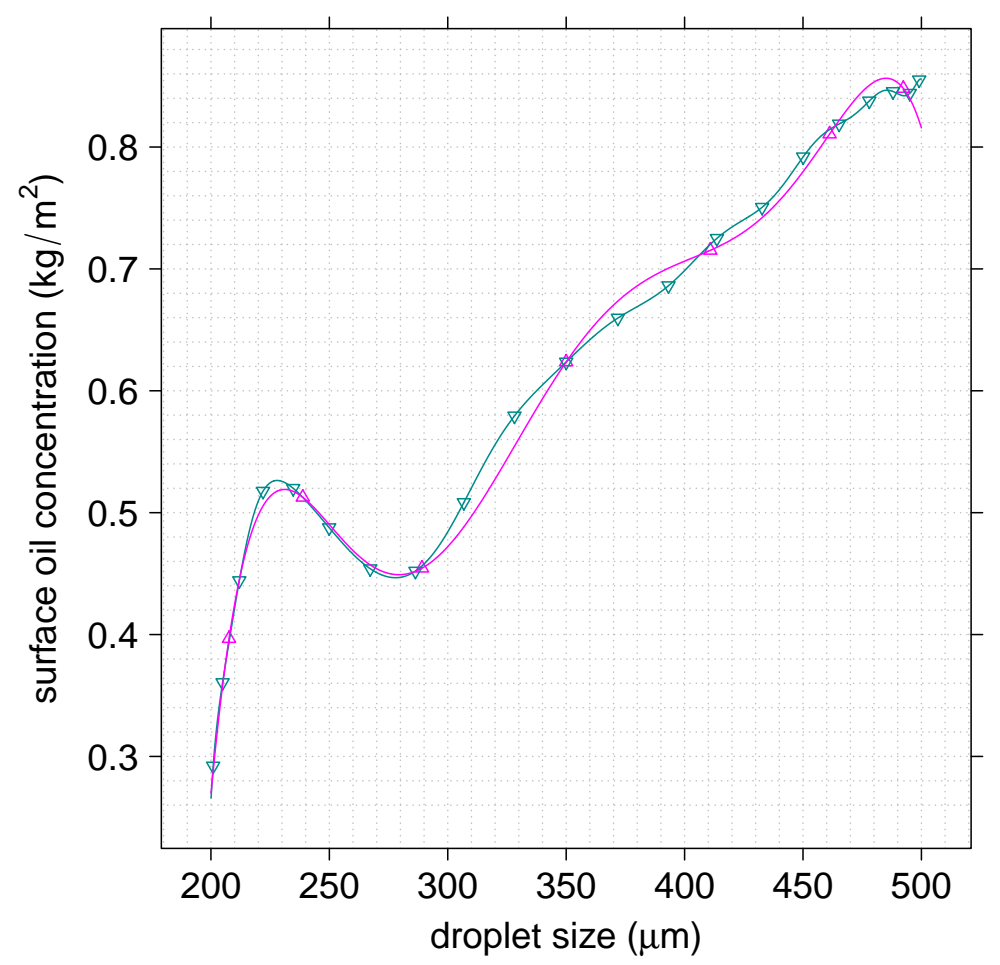

Figure 1: Triangles indicate simulated data at quadrature points based on droplet size being uniformly likely between 200 and $500 \mathrm{~m}$. Curves indicate corresponding polynomial approximations to the response curve. Magenta indicates data and approximation for 6th degree polynomial approximation; cyan, 20th-degree.

specific time after the start of the spill. As uncertainties of many processes ultimately contribute to the uncertainty of the surface concentration, by focusing on droplet size and excluding the rest, we are exploring the uncertainty conditioned on all other inputs, e.g. the advecting velocity field.

Two sets of simulations were available for this study: the first for droplet sizes corresponding to quadrature points for evaluating the coefficients of a series of Legendre polynomials ${ }^{11}$ truncated after the first 7 terms and the second, truncated after the first 21 terms, which approximate the surface concentration of oil using polynomials of 6th-degree and 20th-degree, respec-

\footnotetext{
${ }^{11}$ The Legendre polynomials are appropriate for a uniform probability density. Here droplets are assumed to range from 200 to $500 \mu \mathrm{m}$ in size.
} 
tively. For more details about using quadrature to evaluate coefficients of polynomial expansions, see Appendix B.

Figure 1 illustrates both the quadrature data and the interpolating polynomials. While both approximations have roughly the same shape ${ }^{12}$, they differ in details of the small scales. The magenta curve misses many of the blue triangles, and the cyan curve, although passing through most of the magenta triangles, slightly misses a few for the larger droplet sizes. So it is clear that, even with 21 terms, the polynomial series has not yet converged. This raises the question: how does lack of convergence impact our view of the uncertainty of the surface concentration of the oil in the region of interest? That question will be addressed in the next section.

The maximum near $225 \mu \mathrm{m}$ and minimum near $275 \mu \mathrm{m}$, which are clearly seen in both approximations, indicate a highly nonlinear response and suggest that droplets in different size ranges follow quite different paths from the source to the surface of the target region. In fact, given their widely different behavior, droplets from the three intervals separated by the extrema might be regarded as separate populations and treated separately. Section 4 will show how the existing response curves can be used to examine such possibilities.

\section{Uncertainty propagation via Legendre polynomial expansions.}

Figure 2 shows histograms of the surface oil concentration when droplet size is assumed to be equally likely for all values between 200 and $500 \mu \mathrm{m}$ and

\footnotetext{
${ }^{12}$ This particular output was chosen because of the presence of the relative extrema of its response curve, as that highly nonlinear shape provided a nice example for illustrating the interaction between the input probability density and the response curve in quantifying the uncertainty of the output.
} 

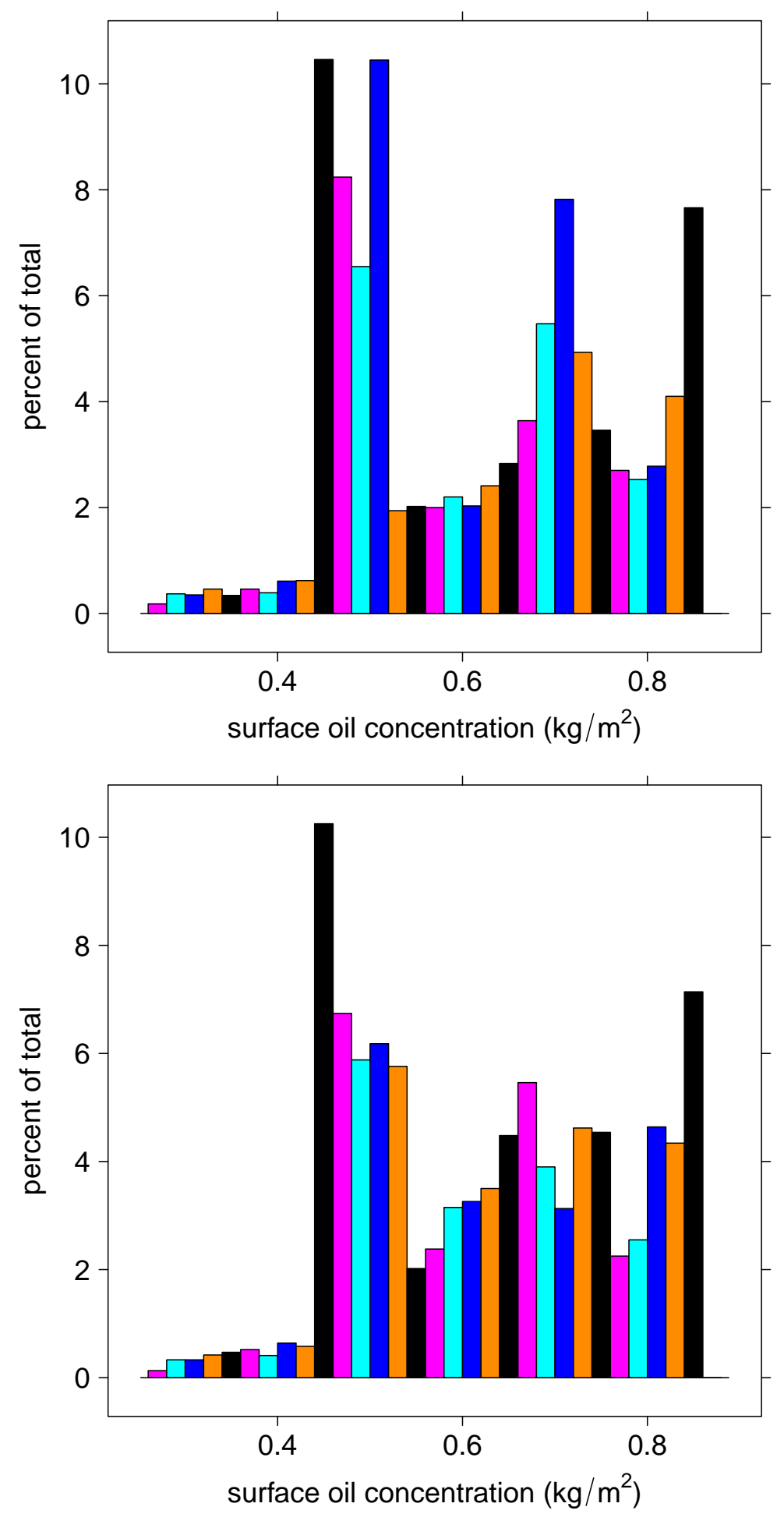

Figure 2: Histograms of surface oil concentration based on 10,000 droplet sizes sampled uniformly from 200 to $500 \mu \mathrm{m}$ have bin width of $0.02 \mathrm{~kg} / \mathrm{m}^{2}$. Upper panel: 6th degree polynomial approximation of response function. Lower panel: 20th- degree approximation. 
completely unlikely for values outside this range. Each histogram bin corresponds to an interval ${ }^{13}$ on the vertical axis of the plot in figure 1 . Projecting that interval onto the response curve and then projecting those portions of the response curve onto the horizontal axis reveals the values of droplet size that contribute to that bin. As the droplet size is uniformly distributed, the height of the histogram for that bin is simply proportional to the sum of the lengths of these projections ${ }^{14}$. For example, consider the bins with the cyan and blue bars near $0.7 \mathrm{~kg} / \mathrm{m}^{2}$ : because the 6th-degree polynomial is considerably flatter in those interval, it has larger projections onto the horizontal axis and thus its histogram has a peak (upper panel) that is noticeably absent when the 20th-degree polynomial is used (lower panel). Clearly, what appear to be relatively minor differences between the 6th-degree and 20thdegree approximations to the response curve induce noticeable differences in the probability density of the response.

The question remains as to the practical significance of these differences. Both histograms indicate a distribution that is far from Gaussian and even multi-modal, and both support the conclusion that the surface oil concentration is highly likely to be greater than $0.44 \mathrm{~kg} / \mathrm{m}^{2}$ but never greater than $0.86 \mathrm{~kg} / \mathrm{m}^{2}$. The greater accuracy of the 20th-degree approximation provided little additional useful information. Using a polynomial of even higher degree

\footnotetext{
${ }^{13}$ To emphasize the relationship between the response curves and the histograms, the horizontal grid lines were chosen to correspond to bin boundaries.

${ }^{14}$ If the response function were monotonic, the output density could be obtained from the input density via a change in scale where the resulting change in shape is associated with stretching or squeezing according to changes in slope for different droplet sizes. But the presence of relative extrema induce a folding in addition to the squeezing and stretching.
} 
would not would not appear to be warranted.

It is important to recognize that this conclusion reflects the assumption that the size of the droplet is confined to be between 200 and $500 \mu \mathrm{m}$ with no preference for one value over another within this range. This assumption of uniformity was made for the convenience of computing the polynomial response using Legendre polynomials. The range simply reflected a droplet size that might be expected with no firm conviction that sizes outside this range were impossible or that all allowed sizes were truly equally likely. Furthermore, as the histogram bins between 0.42 and $0.52 \mathrm{~kg} / \mathrm{m}^{2}$ contain contributions from three different segments of the response curve corresponding to distinctly different paths to the target region, surface concentrations within this range can be expected to be highly sensitive to changing the assumed size distribution to exclude droplets from one or two of the three size ranges. Clearly, the extent to which alternative characterizations of droplet size uncertainty impact the uncertainty of the surface concentration of oil in the target region is worth exploring.

\section{Reconsidering the assumption of uniformity.}

Once the response function has been approximated in a way that is easy to evaluate, it is easy to explore the consequences of alternate characterizations of the input uncertainty. Here we show the consequences of two different distributions of possible droplet size when the surface concentration is approximated by the 20th-degree polynomial discussed above.

Suppose that uncertainty of the droplet size is considerably less than that characterized by the uniform density, which provided the rational for the 

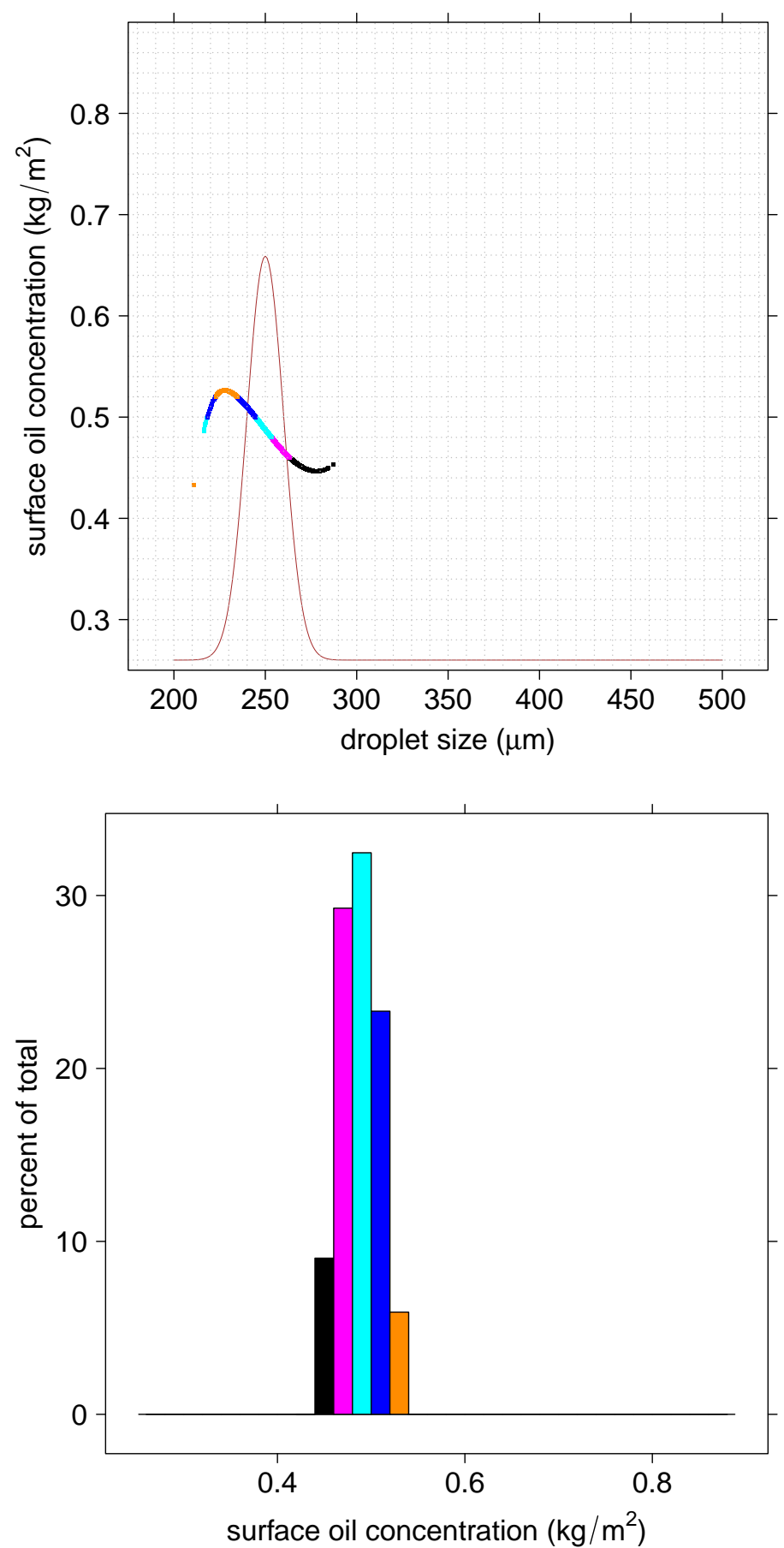

Figure 3: Data from the 20th degree polynomial approximation of response function shown in figure 1. Upper panel: 10,000 approximate response points sampled from a normally distributed droplet-size distribution with mean $250 \mu \mathrm{m}$ and standard deviation $10 \mu \mathrm{m}$ as indicated by the brown curve with unlabeled ordinate. Colors correspond to the histogram bins in the lower panel. Lower panel: Corresponding histogram of possible surface oil concentration in the target region. 
quadrature simulations used to construct the initial approximate responses. For example, assume instead that the input probability density is a Gaussian centered at $250 \mu \mathrm{m}$ with standard deviation of $10 \mu \mathrm{m}$. The upper panel of figure 3 shows this density (scaled to an arbitrary height) and the approximate response for 10,000 droplet sizes randomly drawn from this density. The histogram in the lower panel, as before, reflects the number of samples between each pair of adjacent horizontal grid lines, which have been plotted in different colors so they can be identified more easily. But, as the probability density is no longer uniform, the histogram heights are no longer simple projections of intervals from the response axis onto segments of the response curve and then onto the input axis. Instead, the length of these projections must be multiplied by a factor accounting for the probability of the droplet size being there. Concentrations that project onto the tails of the density clearly contribute negligibly to the histogram. The result is a histogram that is more nearly Gaussian than its predecessor shown in lower panel of figure 2. If the response function were linear, then the probability density for the surface concentration would in fact be Gaussian. But as the response function has a maximum at about $225 \mu \mathrm{m}$ and a minimum at about $275 \mu \mathrm{m}$ - roughly 2.5 standard deviations on either side of the center - the histogram's black and orange bars are a bit longer than would be expected for surface oil concentration described by a Gaussian density.

If this same $\mathrm{N}\left(250,10^{2}\right)$ probability density were propagated using the less accurate 6th-degree polynomial approximation of the response function shown in figure 1, the resulting histogram characterizing the surface concentration would be expected to be much the same, because the two approximate 

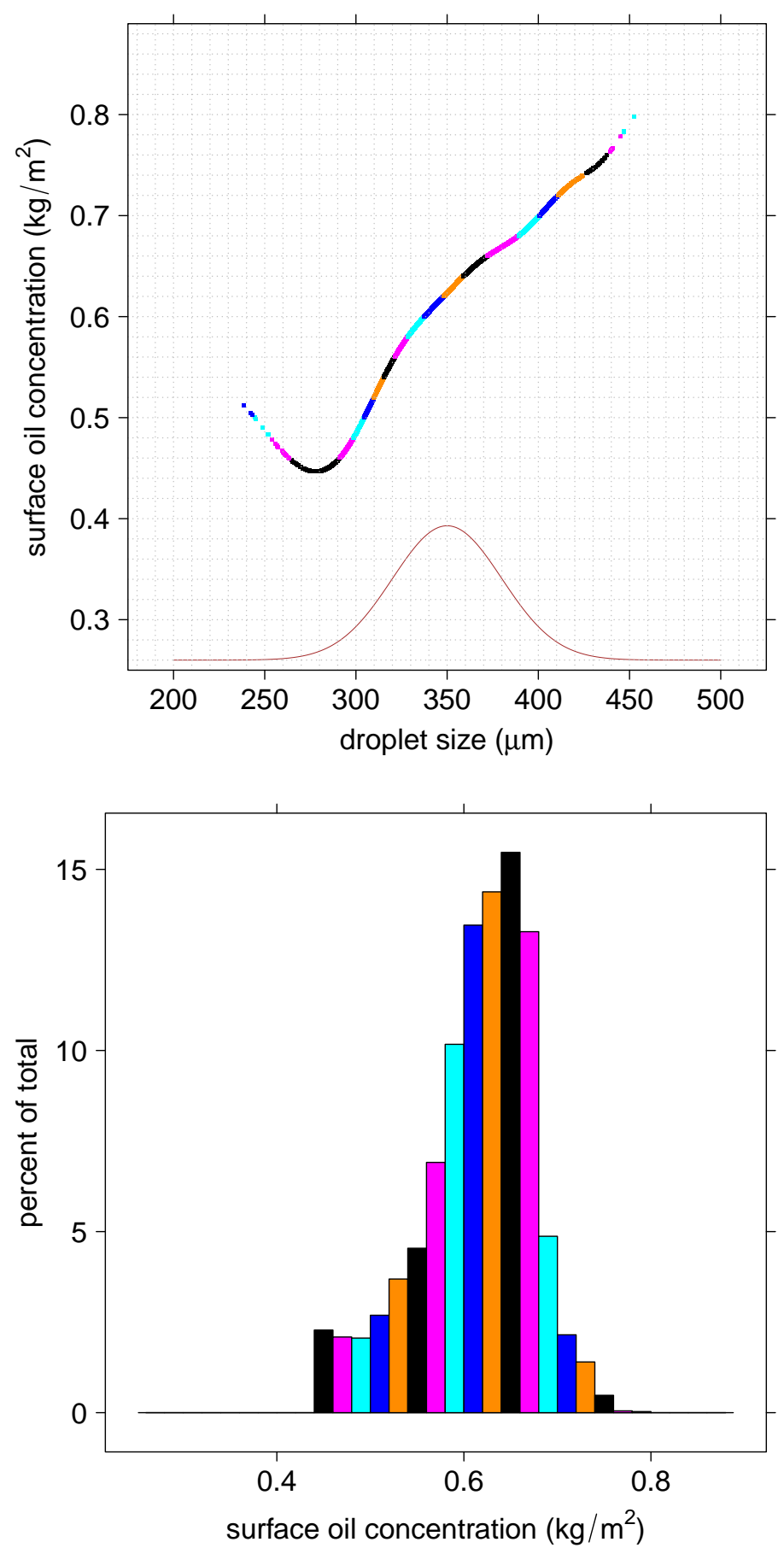

Figure 4: Same as figure 3 except that the possible droplet size is normally distributed with mean $350 \mu \mathrm{m}$ and standard deviation $30 \mu \mathrm{m}$. 
response functions are quite similar for droplet sizes likely to be encountered. For either response curve you could conclude that it would be highly unlikely for the surface concentration to be outside the range of 0.44 to $0.54 \mathrm{~kg} / \mathrm{m}^{2}$ and that the most likely values would be about $5.0 \mathrm{~kg} / \mathrm{m}^{2}$.

Figure 4 shows another example using the 20th-degree polynomial approximation of the response function, this one with droplet size thought to be larger and less certain as described by a Gaussian density centered on $350 \mu \mathrm{m}$ with a standard deviation of $30 \mu \mathrm{m}$. Because the response function is clearly nonlinear over the range of likely valus for droplet size, the resulting histogram reflects a clearly non-Gaussian density. For example, its change in slope near $350 \mu \mathrm{m}$ causes the central orange, black, and magenta bars to be longer than the blue, cyan, and magenta bars to their left. And the local minimum for small values causes the black, magenta, and cyan bars between 0.44 and $0.50 \mathrm{~kg} / \mathrm{m}^{2}$ to be relatively long.

If this wider $\mathrm{N}\left(350,30^{2}\right)$ probability density were propagated using the less accurate 6th-degree polynomial interpolation, its resulting histogram would be different. In particular, the change in its slope near $380 \mu \mathrm{m}$ should cause surface concentrations greater than $0.68 \mathrm{~kg} / \mathrm{m}^{2}$ to be more likely and concentrations less than $0.68 \mathrm{~kg} / \mathrm{m}^{2}$ be less likely, in effect shifting expectations towards higher surface concentrations. Nevertheless both curves would support the conclusion that surface oil concentrations are unlikely to be outside the range of 0.44 to $0.74 \mathrm{~kg} / \mathrm{m}^{2}$ and more likely to be between 0.60 and $0.70 \mathrm{~kg} / \mathrm{m}^{2}$.

The most dramatic sort of revision allows for a significant chance of a droplet being larger (or smaller) than the sizes supporting the presumed uni- 
form density. For example, suppose that the previous density were centered at $450 \mu \mathrm{m}$ rather than $350 \mu \mathrm{m}$, so that a droplet size larger than $500 \mu \mathrm{m}$ must be considered. In such a case the polynomial approximation must be extrapolated beyond the data. Such an extrapolation is likely to provide nonsensical values. For example, figure 1 suggests that the 6th-degree and 20th-degree polynomials would extrapolate rapidly in totally different directions - the former toward smaller concentrations and the latter toward larger concentrations. Obviously, a better approximation to the response function would be needed, which would require additional simulations for larger droplet sizes. Unfortunately, if the same polynomial chaos approach that had been used over the interval from 200 to $500 \mu \mathrm{m}$ were to be used to approximate the response over the enlarged range of droplet size, a new ensemble of quadrature simulations would be needed.

A general conclusion to be drawn from these examples is that lack of knowledge of the probability density characterizing the uncertainty of the input can have an impact on the propagated uncertainty as large as or larger than that resulting from the imprecision of the approximation of the response function. This suggests that the strategy of approximating the response function using quadrature simulations, which are tied to an input density that is likely later to be revised, is an inefficient use of limited computational resources.

\section{Severing the coupling between polynomials and input density.}

The previous section has illustrated that there is no need to stick with the initial view of the relatively poorly known input density: once an approximate 
response curve has been obtained, it can be used to quantify the uncertainty of the response resulting from some other input density. Here we address the possibility of obtaining more flexibility in the choice of simulations by cutting the tie of the polynomial approximation to the input probability density. This requires abandoning the integral expressions for coefficients needed to approximate the response for all possible inputs. Instead of exploiting orthogonality, we can determine the coefficients of a polynomial approximating the response over the range of likely inputs by solving a system of coupled linear equations. Details are provided in Appendix C.

To test this more flexible approach, we combined the two sets of quadrature points, so that we could sample from these without having to compute new simulations. In this way we could explore how well low-degree polynomial approximations determined using likely values of droplet size can characterize the uncertainty of the resulting surface oil concentration. As we have already examined the uncertainties propagated from two different Gaussian input densities, it is instructive to revisit those cases. For each case, 7 simulations centered on the respective density were used to determine a 6th-degree polynomial approximation to a restricted part of the response function.

Figure 5 illustrates the results for the Gaussian density centered at $250 \mu \mathrm{m}$ with standard deviation of $10 \mu \mathrm{m}$. The simulated responses are indicated by triangles, one for the droplet's most likely size, the three next larger sizes, and the three next smaller. Comparing their locations with the brown Gaussian curve reveals that the data span the most likely values. Within the span of the data the gray approximate response curve agrees quite well with the 20th-degree polynomial discussed above, but when extrapolated beyond the 

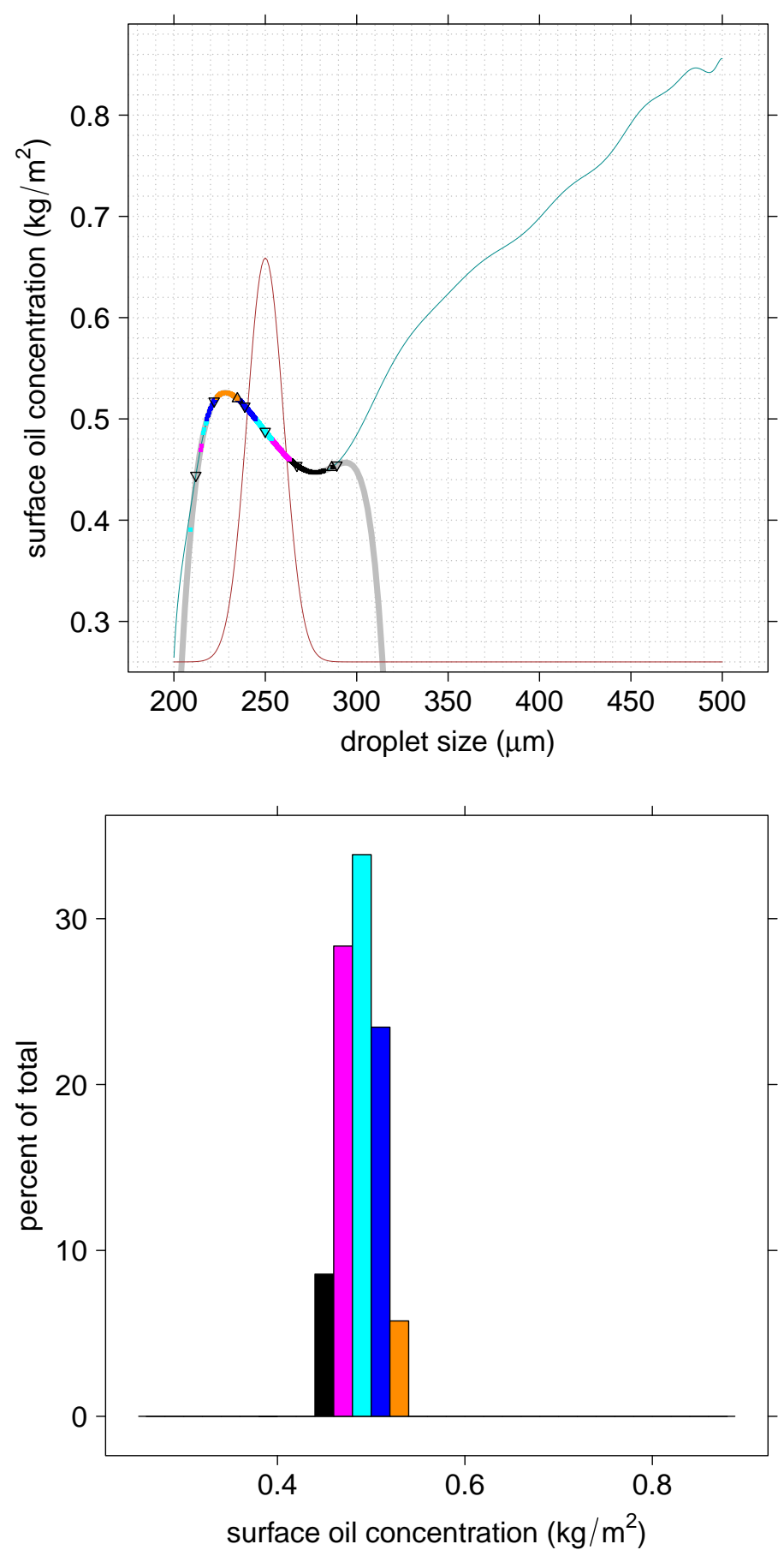

Figure 5: Upper panel: The wide gray curve is the 6th-degree polynomial determined from the data indicated by triangles and the superimposed colored dots indicate the approximate response for each of 10,000 randomly drawn samples from a Gaussian distribution with mean $250 \mu \mathrm{m}$ and standard deviation $10 \mu \mathrm{m}$. The cyan curve is the 20th-degree polynomial from figure 1 and the brown curve indicates the Gaussian density. Lower panel: Corresponding histogram of the 10,000 approximate responses. 
data, this 6th-degree polynomial quickly plunges to nonsensical negative surface concentrations. Because these nonsensical values correspond to droplet sizes that are highly unlikely, they have insignificant impact on the uncertainty of the surface oil concentration in the target region. Consequently, the histogram in the lower panel agrees quite well with its counterpart in figure 3 .

Results for the Gaussian density centered at $350 \mu \mathrm{m}$ with standard deviation of $30 \mu \mathrm{m}$, which are shown in figure 6 , illustrate what happens when the simulations don't quite span the reasonable values of the input. While its ability to interpolate the simulations is excellent, when extrapolating this 6thdegree polynomial suggests unbelievably large surface concentrations. The small fraction of the 10,000 random samples having responses that were extrapolated contribute to a very long tail in the output distribution ${ }^{15}$. But except for this tail of high surface concentration resulting from the extrapolation, the histogram in the lower panel is quite similar to that shown in figure 4.

These examples support the conclusion that when simulations sample the likely values of uncertain inputs, a polynomial approximation of the response function can be sampled to give a good quantitative description of the uncertainty of the output.

\footnotetext{
${ }^{15}$ In fact the plot has been truncated to omit the 27 samples with response greater than $1 \mathrm{~kg} / \mathrm{m}^{2}$, as they comprise a negligible $0.27 \%$ of all samples.
} 

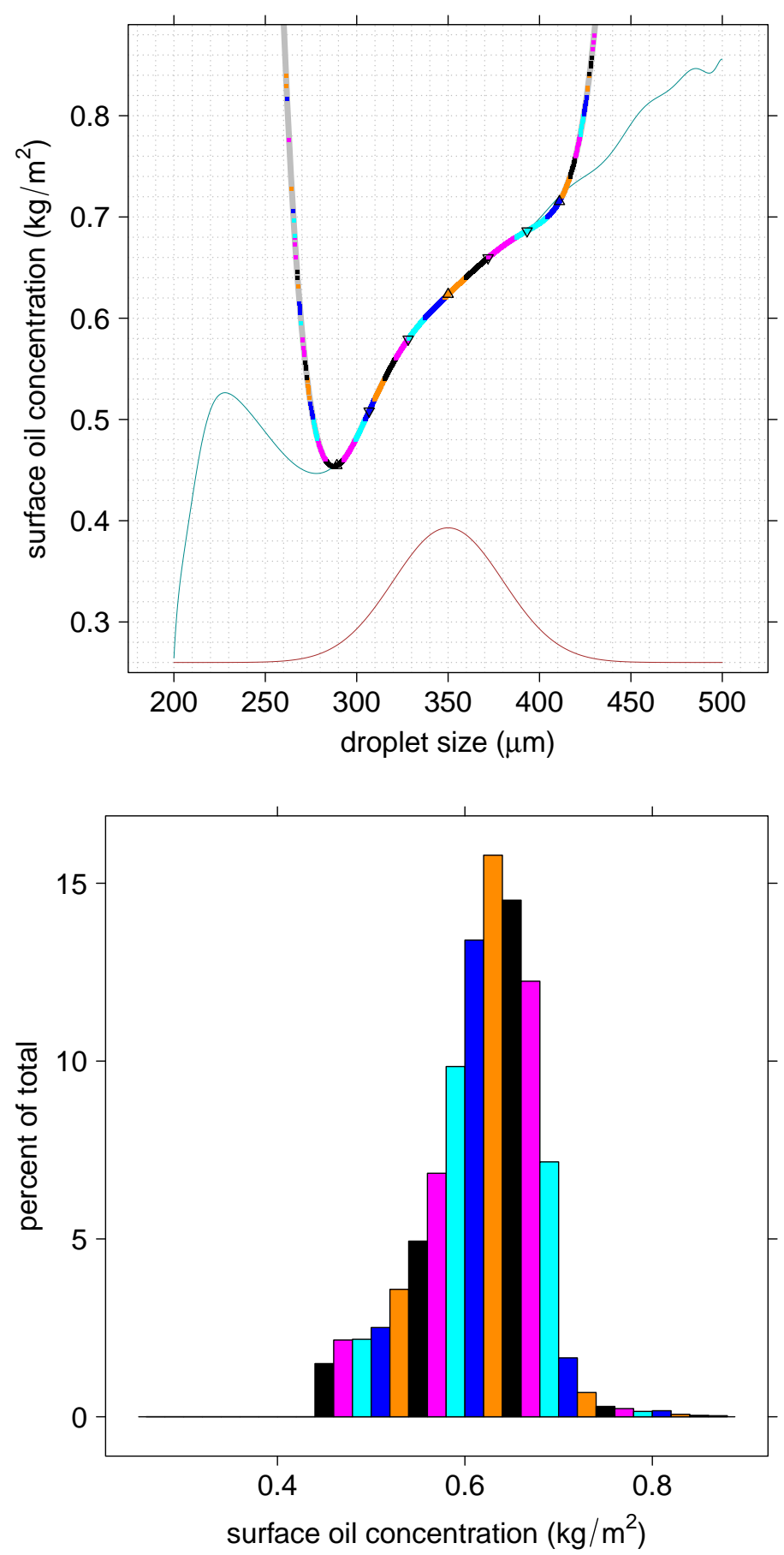

Figure 6: Same as figure 5 except that the possible droplet size is normally distributed with mean $350 \mu \mathrm{m}$ and standard deviation $30 \mu \mathrm{m}$. 


\section{Reconsidering polynomials.}

In this section we illustrate the use of Gaussian processes as an alternative to polynomials for interpolating simulated responses and we explore what consequences this might have on the quantification of the uncertainty of the response. The previous section showed that loosening the tight coupling of polynomials to the input probability density allowed for a more flexible choice of simulations. This flexibility extends to the use of Gaussian processes, as the input density plays no role in their formulation.

A Gaussian process is specified jointly by its mean function, which provides the estimate of the response in the absence of data, and its covariance function, which governs how smoothly the response is expected to change with changing value of the input. Most often the covariance function is taken to be a function of the distance between pairs of values of the input, which returns a small covariance as the separation of the pair gets large. This study uses constant mean functions and squared-exponential covariance functions. For more details, see Appendix D.

Figure 7 shows the results obtained with a Gaussian process using the same seven data points as used for the locally determined 6th-degree polynomial shown in figure 5 . The mean function was taken to be the mean of the seven data: a constant response of $0.49 \mathrm{~kg} / \mathrm{m}^{2}$ surface concentration for all possible values of droplet size. The value of Gaussian process variance parameter was set to $1 \mathrm{~kg}^{2} / \mathrm{m}^{4}$, even though this choice had no impact on the results that are shown ${ }^{16}$. And the length scale of the covariance function was

\footnotetext{
${ }^{16}$ In computing the mean posterior response, the value of the process variance parameter $\sigma_{p}^{2}$ cancels out. For details, see equation(D.5) in Appendix D. However, this choice would
} 

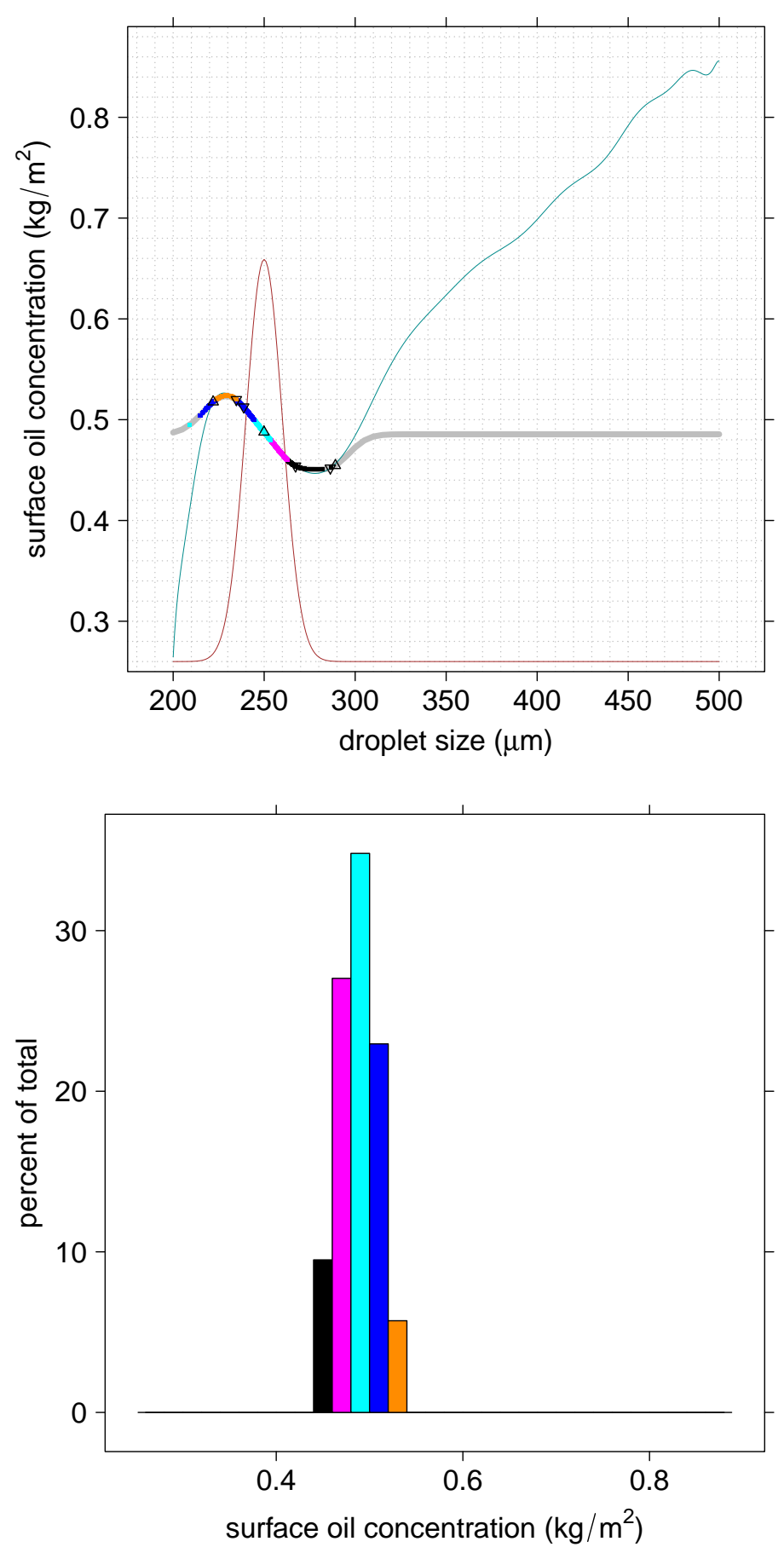

Figure 7: Same as figure 5 except that the response is approximated using a Gaussian process with mean set to the mean of the data and with a covariance function that is a squared exponential with length scale of $10 \mu \mathrm{m}$, which is the same as the standard deviation characterizing the uncertainty of droplet size. 
set at the standard deviation of the $\mathrm{N}\left(250,10^{2}\right)$ probability density accounting for uncertainty in droplet size: $10 \mu \mathrm{m}$. The close agreement between the gray and cyan curves indicates that this choice of length scale provides an excellent approximation to the response curve for any reasonably likely value of droplet size. Note that, when extrapolating beyond the data points, the Gaussian process regresses toward the prior mean, avoiding the unreasonable values that plague polynomial extrapolation ${ }^{17}$. As the Gaussian-process approximation is almost identical to the 6th-degree polynomial approximation of figure 5 within the interval of all reasonably likely values for droplet size, differences being less than the thicknesses of the plotted lines, the histograms of figures 7 and 5 are essentially indistinguishable.

Figure 8 shows a similar comparison of approximate response curves and histograms of Gaussian process and polynomial interpolation using the same data as in figure 6 for the case where the uncertainty in droplet size is $\mathrm{N}\left(350,30^{2}\right)$. Following the pattern of the previous case, the mean function was taken to be the mean of the seven data points: $0.60 \mathrm{~kg} / \mathrm{m}^{2}$; the length scale of the covariance function was set at the standard deviation of the input

be reflected in the posterior covariance function for the Gaussian process and could have been illustrated as an envelope about the gray curve. If added, it would show the envelope decreasing to zero at the data points, increasing between them, and getting quite large as the response curve extrapolates beyond the data. As this choice of $\sigma_{p}^{2}$ was somewhat arbitrary and the plot already complicated, this extra information was not illustrated. On the other hand, if the data were considered to be subject to noise characterized by a variance $\sigma_{n}^{2}$, then the approximate response curve would depend on the ratio $\sigma_{p}^{2} / \sigma_{n}^{2}$ and it would not be required to pass through the data, aand the hyperparameters could be adjusted so that the envelope might provide a more quantitative assment of the accuracy of the interpolation.

${ }^{17}$ Recall that the polynomial interpolation illustrated in figure 5 lead to physically impossible negative values for surface oil concentration. 

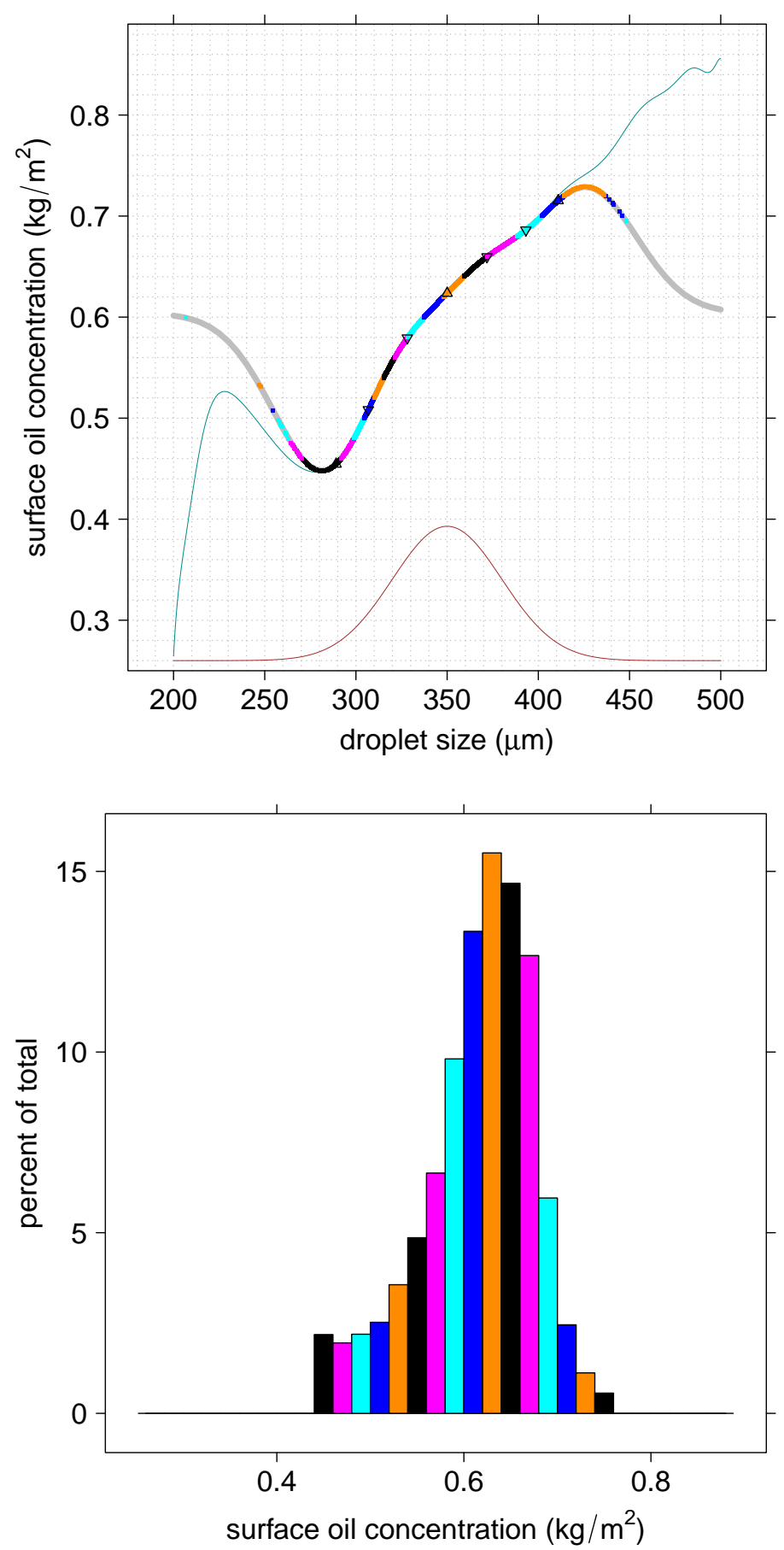

Figure 8: Same as figure 6 except that the response is approximated using a Gaussian process with mean set to the mean of the data and with a covariance function that is a squared exponential with length scale of $30 \mu \mathrm{m}$, which is the same as the standard deviation characterizing the uncertainty of droplet size. 
probability density: $30 \mu \mathrm{m}$; and the variance parameter was set to $1 \mathrm{~kg}^{2} / \mathrm{m}^{4}$. Because the Gaussian process regresses toward the prior mean when extrapolating beyond the data, the histogram describing possible surface oil concentration does not show the heavy tail corresponding to unreasonably high concentrations. Otherwise, given this characterization of the uncertainty of the input, this histogram provides essentially the same information about the uncertainty of the response as those in figures 4 and 6. There is every reason to expect that additional samples from further into the tails of the input distribution might give an excellent approximation to the response curve over the extended range of the data, but given the low probability associated with this extension, the computationally costly simulations providing such samples could not be justified, as they would not substantially impact our understanding of the uncertainty of the surface oil concentration.

\section{Discussion.}

The examples presented here illustrate how the uncertainty of a model's response can be analyzed as an interaction between the shape of its response curve and the probability density of its input. The plots show how colored segments of a response curve are related to the bins of the response histogram and how the probability associated with a particular bin reflects the probability of the input contributing to the corresponding segments. While these examples involve only a single input, the analysis is appropriate for any number of inputs. For example, with two inputs a response surface replaces the response curve and intervals between contours replace segments.

In most practical situations the shape of the response function is un- 
known and the only access to it is via model simulations. While response functions for computationally demanding oceanic and meteorological models cannot be exhaustively sampled, they might be usefully approximated by interpolating a manageable number of simulations. How many simulations are needed is not known in advance and must be determined through exploratory sampling. At the same time, little information is generally available for characterizing input uncertainty, so alternative views should be explored and this influences the simulations that are needed. The expense of each simulation dictates using as few as possible to interpolate the response over the region of likely inputs while adding a few more, as needed, to extend the region to accommodate alternate views of which inputs are likely. It was the lack of flexibility in choice of simulations when using a standard non-intrusive polynomial chaos approach to uncertainty quantification that motivated this study.

The response curve considered here is highly nonlinear: the response first increases, then decreases, and then increases again as the input varies over the explored range, indicating three separate quasi-linear regimes. All inputs within this highly nonlinear range were initially assumed to be equally likely for technical reasons: the range encompassed all reasonable values of the input and the uniform density allowed for the use of sparse quadrature for exploring the impact of several uncertain inputs. The two sets of archived simulations, corresponding to two polynomial approximations of the response curve were ideal for this study, as they could be combined to provide sufficient sampling to explore different interpolations and different views of which input values are likely. The nonlinearity of the response and the uniformity of the 
density resulted in a histogram of possible responses that was multi-modal. Furthermore, the shape of the histogram was sensitive to the accuracy of the interpolation: different views of the smaller-scale wiggles (nonlinearities) of the response curve leading to different assessment of the relative likelihood of possible responses.

Such multi-modal histograms might be expected in a wide range of modeling situations where the range of likely inputs spans a region of highly nonlinear response. It is important to understand that the detailed structure of a response histogram is significant only if there is an important practical question that it can help answer. Given that the analysis is conditioned on the particular model's formulation and on the uncertainties of many other inputs, the individual peaks and valleys of the response histogram are not likely to be sufficiently robust to provide a basis for answering practical questions. This suggests that seeking a more accurate response surface would not be worth the computational effort. The useful information conveyed by these histograms is the possible range of responses resulting from the assumed range of uniformly likely inputs.

On the other hand, different assessments of input uncertainty can have significant practical consequences. By limiting the range of possible inputs, their responses might be confined to quasi-linear sections of the response function. As a linear response function preserves the shape of the density, the response could be expected to be more nearly unimodal with departures from linearity contributing primarily to skewness. This was illustrated by replacing the initial uniform density by two alternative views of input uncertainty, both Gaussian but with different centers and different spreads. 
In most situations little information is available to provide an accurate picture of input uncertainty, and when information does exist transforming it into a probability density can be a lot of work. The process is much like that of data assimilation, as it involves using Bayesian inference to extract information from observations to update a prior view of the density ${ }^{18}$. When accurate information about input uncertainty is unavailable, the only option is to explore the impact of different assumed densities, as illustrated here.

The bulk of this paper treats the technical problem of interpolating the responses, which becomes important when model simulations are limited by their computational expense. The rigidity of the sampling necessitated by the use of quadrature as part of a popular noninvasive polynomial chaos approach for propagating uncertainty motivated our desire for more flexible alternative. We tracked the cause of this rigidity to the exploitation of orthogonality and to the desire to approximate the response globally, both of which stem from the introduction of polynomial chaos by Wiener (1938) before the advent of the computer. We illustrated that orthogonality and quadrature were not essential to the nonintrusive polynomial chaos approach and that the polynomial approximation to the response function could be determined by a standard method for polynomial interpolation. This minor change removes the rigidity and allows the freedom to sample the possible inputs much more flexibly.

Once these parts of the polynomial chaos tradition had been abandoned, it

\footnotetext{
${ }^{18}$ See, for example the discussions of Sraj et al. (2013) and Sraj et al. (2014) on using observations of temperature in the upper ocean to quantify the uncertainty of parameters charactering wind drag on the sea surface.
} 
was clear that all that remained was its use of polynomial interpolation. But what is special about polynomials? Over a small enough interval a smooth function is approximately linear and over a bit larger interval its curvature might be described as quadratic or cubic, but variations over larger regions are likely to require polynomials of higher degree and sufficient data to define them. Gaussian process interpolation was demonstrated as an alternative, as it provides the same sampling flexibility as polynomial interpolation. And through the specification of a prior mean response function it provides a way to avoid unreasonable responses in data voids where interpolation is more like extrapolation and where polynomial interpolation can behave poorly.

One issue that deserves further attention is finding an optimal strategy for choosing which simulations to interpolate. Clearly, when little is known about the response function, the strategy would be exploratory and iterative, using a few simulations to get a first impression and additional simulations as needed and when affordable. When using Gaussian process interpolation, the posterior covariance function could be used to indicate which subsequent simulations might be most effective, as it indicates where the emulator benefit least from the simulations that are already available. Of course, the nature of the variability that is discovered during such an exploration should be used to reassess initial judgment about the choice of the covariance function when using Gaussian process interpolation or the number of terms to retain when using polynomial interpolation.

Another issue deserving attention is the construction of emulators for models incorporating random processes, as repeated simulations using the same inputs produce different responses. Exact interpolation of the simu- 
lated responses is inappropriate and the smoothing capability of regression is needed. Polynomial regression, which is a natural extension of polynomial interpolation when there are more data than the minimum required to determine the coefficients, would allow the response curve to average out the noise. Similarly, Gaussian process regression can smooth out the response through the use of a noise covariance matrix.

A third issue left for the future is identifying a good approach to exploring the uncertainty of multiple inputs. Given the curse of dimensionality, there is a limit to how well the uncertainties of very many inputs can be handled simultaneously. This is an issue that confronts data assimilation, where sampling can never explore all uncertain inputs. Within the context of this paper, there are questions of how many simulations might be needed for for sampling in several dimensions, how they should be chosen, and how to best interpolate them. There is also the question of the relative merit of treating the multiple inputs simultaneously, which is expensive, individually, which is more practical, or pairwise, which is a compromise.

\section{Appendix A. Non-intrusive polynomial chaos.}

Wiener (1938) solved a set of coupled differential equations for the coefficients of the polynomial approximation to the response function. Their derivation is similar to the derivation of the equations for a spectral model of the atmosphere with the polynomial basis being the analogue of the spherical harmonics. ${ }^{19}$. When generalized from his model, which was based on a single

\footnotetext{
${ }^{19}$ Shen et al. (2010) have used this approach to quantify the growth of uncertainty for a Lorentz model.
} 
ordinary differential equation, to models based on systems of coupled partial differential equations, solving for the expansion coefficients has an overhead cost similar to that of constructing a new numerical model, and it is termed intrusive because it requires intimate knowledge of the model's source code. For this reason the intrusive approach is not considered in this paper.

The non-intrusive alternative treats the model as a black box and determines the expansion coefficients by interpolating responses found by running the forecast model multiple times; with $n$ coefficients to be determined, $n$ forecast runs are needed ${ }^{20}$. Here we are interested in the situation where available computational resources limit the number of runs, which in turns limits the number of coefficients that can be determined and thus the degree of the interpolating polynomial. Appendix B and Appendix C provide technical details for two non-intrusive approaches, which differ in how the expansion coefficients are computed.

\section{Appendix B. Determining polynomial coefficients via quadrature.}

The polynomial-chaos approach to propagating uncertainty starts by representing the response function with an infinite series of orthogonal polynomials $P_{j}(x)$, which are chosen based on the probability density $\rho(x)$ describing

\footnotetext{
${ }^{20}$ Webster and Sokolov (2000) have used a non-intrusive polynomial chaos approach for characterizing responses of climate models. Our groups previous work (Thacker et al., 2012; Alexanderian et al., 2012; Sraj et al., 2013; Winokur et al., 2013; Sraj et al., 2014) using a non-intrusive polynomial chaos approach to propagate uncertainties through the hybrid coordinate ocean model (HYCOM) motivated this study.
} 
the uncertainty of the input $x$ :

$$
r(x)=\sum_{j=0}^{\infty} a_{j} P_{j}(x)
$$

549

where $P_{0}(x)=1, P_{1}(x)=x$, and the other polynomials depend on $\rho(x)$ via orthogonality. The expression for the orthogonality of the polynomials $P_{n}(x)$ is:

$$
\int P_{j}(x) P_{k}(x) \rho(x) d x=\delta_{j, k} N_{k}
$$

where the integral extends over the entire support of the input density $\rho(x)$, where $N_{k}$ is a normalization constant, and where $\delta_{j, k}$ is the Kronecker delta. Multiplying the response function by $P_{k}(x) \rho(x)$ and integrating thus yields an expression for each coefficient:

$$
a_{k}=\frac{1}{N_{k}} \int r(x) P_{k}(x) \rho(x) d x
$$

If the response function is sufficiently smooth, it can be approximated with a polynomial of degree $n$ by truncating the expansion:

$$
\hat{r}(x)=\sum_{j=0}^{n} a_{j} P_{j}(x) .
$$

In the two examples of figure 1, where the input density is uniform and the expansion is in Legendre polynomials, one curve corresponds to $n=6$ and the other to $n=20$.

As the response function is expensive to evaluate, the strategy is to approximate the integrals for the coefficients in (B.3) by efficient quadrature 
formulae using the values of the response evaluated for only a few values $x_{q}$. In this way the integral becomes a sum over an ensemble of quadrature points with the corresponding quadrature weight $w_{q}$ appearing as a factor in each term, so that:

$$
a_{k} \approx \frac{1}{N_{k}} \sum_{q} r\left(x_{q}\right) P_{k}\left(x_{q}\right) w_{q} .
$$

For Gaussian quadrature, the quadrature ensemble and weights are chosen so that using $n+1$ points yields the exact integral whenever the integrand is a polynomial of degree $2 n$ or less ${ }^{21}$. So using $n+1$ quadrature points to evaluate all coefficients $a_{1}, \ldots, a_{n}$ guarantees that the approximate response function will be a polynomial passing through each of the quadrature points. As the integral in (B.3) extends over the entire support of the input density $\rho$, i.e. over all possible values of the input and the coefficients guarantee global polynomial exactness, the quadrature points tend to accumulate far from the center of the region of highly likely inputs. Furthermore, the locations of the quadrature points depend sensitively on the input density $\rho$, which is poorly known. Given the expense of the quadrature simulations, it might be wise to seek an approximation to the response function that is less strongly tied to the input density and that is less concerned with global polynomial exactness.

Once the coefficients have been evaluated, multiplying appropriate expressions involving $\hat{r}(x)$ by $\rho(x)$ and integrating yields expressions for the statistical moments of the response. For example, because all of the polynomials except for the constant average to zero, $\overline{\hat{r}}=a_{0}$, and because the

\footnotetext{
${ }^{21}$ See, e.g., Abramowitz and Stegun (1970) or Davis and Rabinowitz (2007).
} 
polynomials are orthogonal, $\overline{(r-\overline{\hat{r}})^{2}}=\sum_{j=1}^{n} a_{j}^{2} N_{j}$. Such expression for the moments can provide a useful and inexpensive characterization of the uncertainty of the response — but only if $\rho(x)$ provides an adequate description of the input uncertainty.

A general feature of the quadrature ensembles used to build surrogates is that they include simulations for inputs that are significantly different than the default values that gave rise to the forecasts. In fact, some of the quadrature simulations might fail, because the model cannot handle such extreme inputs. This is the consequence of the expressions for the polynomial coefficients, which require integrating over all possible inputs, however unlikely, together with quadrature attempting to guarantee polynomial exactness. In other words, it is a result of the global way in which the problem has been formulated. As a result, the tails of a poorly known approximate input distribution can play a much larger role than they should in the design of the quadrature ensemble of model runs that comprise the overwhelming bulk of the computation effort. An alternative to quadrature, which allows the ensemble to be chosen more flexibly, is described in Appendix C.

\section{Appendix C. Determining polynomial coefficients by solving a linear system of equations.}

The polynomial-chaos strategy of polynomial interpolation does not require that the polynomial coefficients be determined by quadrature (B.5). A straight-forward alternative is to solve for the coefficients algebraically. Rather than using simulated responses to evaluate an expression based on the orthogonality of the polynomials (B.3), just solve an algebraic system 
that expresses the fact that the polynomial passes through the simulations. As this alternative does not exploit orthogonality of the terms comprising the polynomial, it lessens the bond between the approximating polynomial and the probability density describing the uncertainty of the input and allows the simulations to be chosen more flexibly. When they are chosen at the quadrature points, the polynomial response curve is exactly the same as you would get using quadrature. But if they are chosen to focus the accuracy more locally, the different coefficients can be expected and remote parts of the response curve might differ substantially.

While the response can be represented as a sum of orthogonal polynomial terms, since orthogonality is not exploited, this is not necessary. Instead, it can be represented simply in terms of powers of the input. If the response is to be approximated by a polynomial of degree $n$ :

$$
\hat{r}(x)=c_{0}+c_{1} x+c_{2} x^{2}+\ldots+c_{n} x^{n},
$$

then its coefficients $c_{j}, j=0, \ldots, n$ can be determined from $n+1$ simulated responses $r_{i}, i=1, \ldots, n+1$ to inputs $x_{i}, i=1, \ldots, n+1$ by solving a system of linear equations:

$$
\mathbf{r}=\mathrm{Dc},
$$

stating that the vector of responses $\mathbf{r}$ is the product of the vector of coefficients $\mathbf{c}$ and a design matrix ${ }^{22} \mathbf{D}$, each column of which corresponds to a

${ }^{22}$ Within the context of polynomial interpolation, this matrix is often referred to as the Vandermonde matrix. Design matrix is terminology most often used in the context of regression modeling where there are more data than unknowns. In that case the polynomial would not be expected to agree with all the data, so an additional term is added to the 
power of the input and each row to a simulated response ${ }^{23}$.

The solution for the coefficients $\mathbf{c}=\mathbf{D}^{-1} \mathbf{r}$ requires $\mathbf{D}$ to be invertible. In other words, the simulations must be chosen so that they are able to distinguish between the different powers of the input. While this was not an issue for the simulations used in our examples, it is something that deserves attention. Fortunately, the design matrix can be examined before any simulations are computed to insure that the planned simulations correspond to a well-conditioned design matrix.

Solving a linear system for the polynomial's coefficients does not preclude the possibility of obtaining simple expressions for statistics of the response. If the input probability density $\rho$ can be approximated by a density that serves as an orthogonality weight for a set of orthogonal polynomials, then it is quite straightforward to re-express the powers of the input in (C.1) as linear combinations of those polynomials in order to find the coefficients in which the statistics are expressed. In the examples of figures 5 and 6 , which are based on Gaussian densities, Hermite polynomials would be appropriate.

Because the more flexible approach to computing the polynomial coefficients is not based on the integral expressions, which were derived by exploiting the orthogonality of the polynomials, the strong coupling between the polynomial approximation of the response function and the assumed in-

right-hand side of (C.1) to characterize the misfit, and the statistics of the misfit can be used to quantify the error of the polynomial fit to the data. The least-squares coefficients would be given by $\mathbf{c}=\left(\mathbf{D}^{T} \mathbf{D}\right)^{-1} \mathbf{D}^{T} \mathbf{r}$ rather than by $\mathbf{c}=\mathbf{D}^{-1} \mathbf{r}$.

${ }^{23}$ If the polynomial approximation had been expressed as a sum of orthogonal polynomials rather than simply powers of the inputs, then the columns of the design matrix would be the polynomials evaluated at the values of the inputs and the coefficient vector would contain the coefficients $a_{k}$ used for the polynomial chaos expansion. 
put density is broken. Most of the polynomial chaos formalism is no longer needed. All that remains is the fact that the response is approximated as being polynomial.

The next step is to consider other approximations. An obvious choice is Gaussian process interpolation, because it limits the influence of the simulated responses to the region of likely values without trying to extrapolate to the tails of poorly determined input densities. Appendix D describes how it works.

\section{Appendix D. Gaussian processes.}

Gaussian process regression is just another term for objective mapping or optimal interpolation where point observations are used to approximate continuous fields, and Gaussian process interpolation is just the limit where the observations are treated as being noise-free. Within our current context, simulations provide the counterparts of the data that are to be optimally interpolated and the response function is the counterpart of the fields that are to be corrected ${ }^{24}$.

Gaussian process interpolation is conceptually quite different from polynomial interpolation: its error is described statistically rather than being relegated to the discarded terms of an infinite series, and the choice of its statistical description replaces the choice of the interpolating polynomial's degree. Furthermore, the statistics are best regarded as Bayesian. The process is defined by a mean function $m(x)$ and a covariance function $K\left(x, x^{\prime}\right)$,

\footnotetext{
${ }^{24}$ For an overview of Gaussian processes, see Rasmussen and Williams (2006) and for a discussion of optimal interpolation see Daley (1993).
} 
which provide the prior statistical description. The data to be interpolated are used to update the prior with the resulting posterior mean providing the interpolating function. The posterior covariance function reflects the interpolating function's exact agreement with the data as well as the error away from the data ${ }^{25}$.

The interpolated response $\hat{r}(x)$ for any possible input $x$ is the posterior mean of the Gaussian process after taking into account the information provided by the simulations. It can be regarded as a correction to the prior mean that can be computed as a linear combination of the corrections needed to make the prior mean function agree with the simulated responses $r_{j}$ for inputs $x_{j}$ :

$$
\hat{r}(x)-m(x)=\sum_{j=1}^{n} \alpha_{j}(x)\left(r\left(x_{j}\right)-m\left(x_{j}\right)\right),
$$

where the differences $r\left(x_{j}\right)-m\left(x_{j}\right)$ are often called innovations. In this paper the mean function is taken to be a constant and set to the mean of the simulated responses. The coefficients $\alpha_{j}$ that define the liner combination are determined by the covariance function $K\left(x^{\prime}, x^{\prime \prime}\right)$ of the Gaussian process, which relates responses for all pairs of inputs $x^{\prime}$ and $x^{\prime \prime}$. Typically, a covariance function is chosen to be a function of the distance $\left|x^{\prime}-x^{\prime \prime}\right|$ between the input pairs and, even then, a wide variety of different function can be

${ }^{25}$ While the posterior covariance function can also be used to measure the error of the interpolation away from the data, this measure only reflects the choice for the prior covariance function, which imposes a view of the smoothness in much the same way as truncation of an infinite series would. It should be regarded not as a quantitative measure of interpolation error but as a qualitative indication of the limits of the influence of the data given the assumptions of correlation length. Error envelopes could have been added to the plots in figures 5 and 6 , but would not have added significantly to the discussion. 
used. Here we restrict our attention to the squared exponential covariance function:

$$
K\left(x^{\prime}, x^{\prime \prime}\right)=\sigma_{p}^{2} \exp \left(-\frac{\left|x^{\prime}-x^{\prime \prime}\right|^{2}}{2 \Delta^{2}}\right),
$$

where $\sigma_{p}^{2}$ is a constant variance and $\Delta$ scales the distance.

Central to the computation of the linear combinations is the $n \times n$ covariance matrix $\mathbf{K}$ relating the $n$ simulations that are to be interpolated:

$$
\mathbf{K}_{i, j}=K\left(x_{i}, x_{j}\right) .
$$

Its inverse converts the elements or the innovation vector $\mathbf{r}-\mathbf{m}$ into a vector $\delta=\mathbf{K}^{-1}(\mathbf{r}-\mathbf{m})$ having components that represent the relative influence of each of these $n$ simulations. For any input $x$ the response is approximated by weighting $\delta_{j}$ by the covariances $K\left(x, x_{j}\right)$ and then summing over the simulations. If interpolated values are desired for $N$ inputs $x_{k}$ where simulations may or may not be available, then it is useful to introduce the $N \times n$ covariance matrix relating these inputs to those where simulation are available:

$$
\mathbf{K}_{k, j}^{*}=K\left(x_{k}, x_{j}\right) .
$$

Then the vector of $N$ interpolated responses $\hat{\mathbf{r}}$ can be written compactly as:

$$
\hat{\mathbf{r}}=\hat{\mathbf{m}}+\mathbf{K}^{*} \mathbf{K}^{-1}(\mathbf{r}-\mathbf{m})
$$

where vector $\hat{\mathbf{m}}$ contains the estimated mean responses before interpolation, i.e., the prior mean given by the mean function of the Gaussian process evaluated at the target points of the interpolation, and the second term is 
the correction provided by the simulations.

Note that the predicted responses do not depend on the magnitude of the covariance function but only on its shape, as the variance $\sigma_{p}^{2}$ cancels out in equation (D.5) ${ }^{26}$. Note also that, when there are the same number of target points as simulations and when those points are chosen to coincide with the inputs of the simulations, $\mathbf{K}^{*}$ becomes identical to $\mathbf{K}$ and $\hat{\mathbf{m}}$ to $\mathbf{m}$, so the interpolating function passes through all of the simulated values. In other words, the prior variance $\sigma_{p}^{2}$, is replaced by a posterior variance of zero.

Just as the simulations provide information for replacing the Gaussian process's mean function with a posterior mean function that can be evaluated pointwise, they also provide a posterior covariance function that indicates zero variance at the simulations and reduced variance at interpolated values. For the $N$ interpolated points the elements of the $N \times N$ prior covariance matrix $\mathbf{K}^{\dagger}$ are just the Gaussian process covariance function evaluated at for pairs of target points $\mathbf{K}_{k \cdot k^{\prime}}^{\dagger}=K\left(x_{k}, x_{k^{\prime}}\right)$. The posterior covariance matrix:

$$
\hat{\mathbf{K}}^{\dagger}=\mathbf{K}^{\dagger}-\mathbf{K}^{*} \mathbf{K}^{-1} \mathbf{K}^{*}
$$

reflects a reduced uncertainty due to the information provided by the simulations. When the target points are the same as the inputs for the simulations, the poster covariance matrix is zero, confirming that the posterior mean function reproduces the simulations. For target points that are very close to the simulations, the covariance matrix doesn't quite vanish, but its diagonal

\footnotetext{
${ }^{26}$ In the limit of $\sigma_{p}^{2} \rightarrow 0$, where this cancellation may be problematic, the covariance function (D.2) no longer makes sense.
} 
elements are small, indicating a reduction in variance in the neighborhood of the simulations. But at points far enough from the simulations for prior correlations to be tiny, its diagonal elements, i.e. the posterior variances, are approximately the same as the prior variance.

If the covariance function were a true characterization of uncertainty of what the model might produce at the target points for interpolation, then the posterior covariances would provide an estimate of interpolation error. But this is not the case. The covariance function should be regarded simply as a prescription for interpolating and its choice is analogous to the choice of where to truncate the polynomial series. Just as the posterior mean function, which prescribes the interpolation, is independent of the scaling of the prior variance, the reduction in variance shown in (D.6) is proportional to $\sigma_{p}^{2}$. A large $\sigma_{p}^{2}$ would show greater reduction in variance than a small one, but without information about how to set its size, what to use is arbitrary. So error estimates based on the posterior covariances provide only a qualitative indication of interpolated values that have benefited little from the information provided by the simulations.

Because this paper addresses the issue of propagating uncertainty through computationally demanding numerical models, the emphasis has been on using as few simulations as possible to provide inexpensive emulators. Discussion of regression-based approaches did not seem appropriate, as they require more simulations for approximating the response function. However, in some cases regression might be justified. For example, the model might contain stochastic processes and give different results when run repeatedly for the same inputs. In that case the desired response is a function that filters the 
stochastic noise much as a straight line provides a useful summary of data presented as a scatter plot. Even without stochastic processes the model might involve iterations with convergence criteria chosen to provide reasonable results with few iterations. In that case the number of iterations might change as an input parameter is varied causing the response function to appear noisy. Again, some filtering is desirable. Another motivation might be to accommodate the fact that the numerical model is inexact. But whatever the reason, the response is desired to be smoother than the simulated data. This mention of regression has been added in response to a question about the possibility of using the posterior variance of a Gaussian process to characterize interpolation error. Just as the standard deviation of the scatter of data about a regression line provides an envelope within which data are most likely to fall, the same is true for Gaussian process regression. And just as the noise variance is not know before building the linear regression model but is determined after fitting to the data, the hyperparameters of the Gaussian process can be adjusted to make its error envelope meaningful.

When data have noise centered about posterior mean of the Gaussian process with covariance matrix $\boldsymbol{\Sigma}_{n}=\sigma_{n}^{2} \mathbf{I}$, then the posterior mean function is not given by (D.5) but by:

$$
\hat{\mathbf{r}}=\hat{\mathbf{m}}+\mathbf{K}^{*}\left(\mathbf{K}+\boldsymbol{\Sigma}_{n}\right)^{-1}(\mathbf{r}-\mathbf{m}),
$$

so the posterior mean function for Gaussian process regression in not independent of the value of $\sigma_{p}^{2}$ and it does not pass through the simulated responses. Similarly, the expression for the posterior covariance function 
771

(D.6) is replaced by:

$$
\hat{\mathbf{K}}^{\dagger}=\mathbf{K}^{\dagger}-\mathbf{K}^{*}\left(\mathbf{K}+\boldsymbol{\Sigma}_{n}\right)^{-1} \mathbf{K}^{*}
$$

772

The hyperparameters for variance $\sigma_{p}^{2}, \sigma_{n}^{2}$ and correlation length $\Delta$ can be adjusted, e.g., by cross-validation, to obtain an error envelope encompassing the bulk of the data.

\section{Acknowledgments}

This research was supported by the Office of Naval Research, Award N00014-101-0498 and Department Research Initiative (DRI) ITOP grant N00014-08-1-0576, by the US Department of Energy, Office of Advanced Scientific Computing Research, Award Number DE-SC0007020, by the Gulf of Mexico Research Initiative contract numbers SA1207GOMRI005 (CARTHE) and SA12GOMRI008 (DEEP-C), and by King Abdullah Univerity Science and Technology contract number KAUST-2014-C0378.

\section{References}

Abramowitz, M., Stegun, I., 1970. Handbook of Mathematical Functions. Dover.

Alexanderian, A., Winokur, J., Sraj, I., Srinivasan, A., Iskandarani, M., Thacker, W. C., Knio, O., 2012. Global sensitivity analysis in an ocean general circulation model: a sparse spectral projection approach. Comput. Geosci. 16, 757-778, doi:10.1007/s10596-012-9286-2.

Borgonovo, E., Castaings, W., Tarantola, S., Jun. 2012. Model emulation and moment-independent sensitivity analysis: An application to environmental modelling. Environ. Model. Softw. 34, 105-115, doi:10.1016/j.envsoft.2011.06.0 06. 
Castaings, W., Borgonovo, E., Morris, M. D., Tarantola, S., 2012. Sampling strategies in density-based sensitivity analysis. Environ. Model. Softw. 38, 13-26, doi:10.1016/j.envsoft.2012.04.017.

Daley, R., 1993. Atmospheric Data Analysis. Cambridge University Press, Cambridge.

Davis, P. J., Rabinowitz, P., 2007. Methods of Numerical Integration, 2nd Edition. Dover.

Eldred, M. S., Webster, C. G., Constantine, P. G., 2008. Evaluation of non-intrusive approaches for Wiener-Askey generalized polynomial chaos. Proceedings of the 10th AIAA Non-Deterministic Approaches Conference, number AIAA-2008-1892, Schaumburg, IL 117, 189.

French, S., 2003. Modelling, making inferences and making decisions: The roles of sensitivity analysis. TOP 11, 229-251.

Kennedy, M. C., O'Hagan, A., 2000. Predicting the output from a complex computer code when fast approximations are available. Biometrika 87 (1), $1-13$.

Najm, H. N., 2009. Uncertainty quantification and polynomial chaos techniques in computational fluid dynamics. Annu. Rev. Fluid Mech. 41, 35-52.

Rasmussen, C. E., Williams, C. K., 2006. Gaussian Processes for Machine Learning. MIT Press, Cambridge.

Ratto, M., Pagano, A., Young, P., 2009. Non-parametric estimation of conditional moments for sensitivity analysis. Reliab. Eng. Syst. Saf. 94, 237-243, doi:10.1016/j.ress.2008.02.023.

Rougier, J., Sexton, D. M. H., Murphy, J. M., Stainforth, D., 2009. Analyzing the climate sensitivity of the HadSM3 climate model using ensembles from different but related experiments. Journal of Climate 22 (13), 3540-3557.

Sacks, J., Welch, W. J., Mitchell, T. J., Wynn, H. P., 1989. Design and analysis of computer experiments. Stat. Sci. 4 (4), 409-435.

Shen, C. Y., Evans, T. E., Finette, S., 2010. Polynomial chaos quantification of the growth of uncertainty investigated with a Lorenz model. J. Atmos. Oceanic Technol. 27, 1059-1071, doi:10.1175/2009JTECHO727.1. 
Sraj, I., Iskandarani, M., Srinivasan, A., Thacker, W. C., Winokur, J., Alexanderian, A., Lee, C.-Y., Chen, S. S., Knio, O. M., 2013. Bayesian inference of drag parameters using AXBT data from Typhoon Fanapi. Mon. Wea. Rev. 141 (7), 2347-2366, doi:10.1175/MWR-D-12-00228.1.

Sraj, I., Iskandarani, M., Thacker, W. C., Srinivasan, A., Knio, O. M., 2014. Drag parameter estimation using gradients and Hessian from a polynomial chaos model surrogate. Mon. Wea. Rev. 142 (2), 933-941, doi:10.1175/MWR-D-13-00087.1.

Thacker, W. C., Srinivasan, A., Iskandarani, M., Knio, O. M., Henaff, M. L., 2012. Propagating boundary uncertainties using polynomial expansions. Ocean Model. 43-44, 52-63, doi:10.1016/j.ocemod.2011.11.011.

Webster, M. D., Sokolov, A. P., 2000. A methodology for quantifying uncertainty in climate projections. Climate Change 46, 417-446, doi:10.1023/A:1005685317358.

Wiener, N., 1938. The homogenous chaos. Am. J. Math. 60, 897-936.

Winokur, J., Conrad, P. P., Sraj, I., Knio, O. M., Srinivasan, A., Thacker, W. C., Marzouk, Y., Iskandarani, M., 2013. A priori testing of sparse adaptive polynomial chaos expansions using an ocean general circulation model database. Comput. Geosci. 17, 899-911, doi:10.1007/s10596-0139361-3.

Xiu, D., 2009. Fast numerical methods for stochastic computations: A review. Commun. Comput. Phys 5, 242-272.

Yang, J., 2011. Convergence and uncertainty analyses in monte-carlo based sensitivity analysis. Environ. Model. Softw. 26 (4), 444-457, doi:10.1016/j.envsoft.2010.10.007. 